\title{
POSITIVE COMBINATIONS OF PROJECTIONS IN VON NEUMANN ALGEBRAS AND PURELY INFINITE SIMPLE C*-ALGEBRAS
}

\author{
VICTOR KAFTAL, P. W. NG, AND SHUANG ZHANG
}

\begin{abstract}
We give an overview of the question: which positive elements in an operator algebra can be written as a linear combination of projections with positive coefficients. A special case of independent interest is the question of which positive elements can be written as a sum of finitely many projections. We focus on von Neumann algebras, on purely infinite simple $\mathrm{C}^{*}$-algebras, and on their associated multiplier algebras.
\end{abstract}

\section{Introduction and preliminaries on projections in $\mathbf{C}^{*}$-algebras}

In this article we focus on a number of questions involving projections in $\mathrm{C}^{*}$ algebras.

Question 1.1. Which positive elements in a $C^{*}$-algebra $\mathcal{A}$ are linear combination of projections with positive coefficients? (Positive combinations of projections for short.) And for which algebras are all positive elements positive combinations of projections?

A case of particular interest is when all the coefficients are equal to one.

Question 1.2. Which positive elements in a $C^{*}$-algebra $\mathcal{A}$ are finite sums of projections?

A related and somewhat simpler question for those $\mathrm{C}^{*}$-algebras where it is natural to consider also infinite sums (e.g., von Neumann algebras, with convergence in the strong topology or multiplier algebras of a $\mathrm{C}^{*}$-algebra with convergence in the strict topology) is the following:

Question 1.3. Which positive operators in a $C^{*}$-algebra $\mathcal{A}$ are finite or infinite sums of projections?

We will study these questions for von Neumann algebras, for $\sigma$-unital purely infinite simple $\mathrm{C}^{*}$-algebras and for their associated multiplier algebras. In the future, we plan to extend our analysis to a wider class of algebras.

Our paper is organized as follows. In Section 2, we review some of the basic notions related to projections in $\mathrm{C}^{*}$-algebras.

In Section 3 we survey known results related to linear combination of projections. We introduce formally an algebra constant $v_{0}$ that bounds the minimal sum of the moduli of the coefficients (see Definition 3.1). To the best of our knowledge, this constant was first introduced for $\mathbb{B}(\mathcal{H})$ by Fong and Murphy in 13. Although often not explicitly referred to, this constant is implicit in much of the work on linear combinations of projections. By combining the work of Fack [9] and Marcoux 
26] we show the existence of that constant for a large class of $\mathrm{C}^{*}$-algebras (see Proposition (3.9). The reason that we are interested in the existence of $v_{0}$ is that together with an additional mild condition, it guarantees that positive invertible elements are positive combinations of projections (see Proposition 4.2).

In Section 4 we review the results about positive combinations of projections in $\mathbb{B}(\mathcal{H})$ and then present a simple but useful lemma (Lemma 4.3) that is the key to decomposing positive elements into positive combinations of projections. We apply it to three kinds of $\mathrm{C}^{*}$-algebras.

For von Neumann algebras, it permits us to obtain a sufficient condition in terms of spectral resolution. This condition is also necessary if the range projection of the element is properly infinite and this condition is always satisfied for finite factors and $\sigma$-finite type III factors (Theorem 4.4). For factors, this condition is equivalent to the non-vanishing of the essential norm with respect to the relative compact ideal (i.e., the ideal generated by the finite projections of the factor).

For global von Neumann algebras, a similar condition (see Theorem 4.6) holds by replacing essential norm with central essential norm and invoking the theory of central ideals of Halpern [15, [16] and Stratila and Zsido 33] (see Section 4 for these notions).

One might argue that $\sigma$-unital purely infinite simple $\mathrm{C}^{*}$-algebras share some properties with $\sigma$-finite type III factors. The analogy holds in this case: all positive elements in either type of algebras are positive combinations of projections (Theorems 4.4(iii), 4.7). Moreover, the same holds for multiplier algebras of $\sigma$-unital purely infinite simple $\mathrm{C}^{*}$-algebras (Theorem 4.8).

In Section 5, we discuss decompositions into infinite sums of projections. Their characterization is complete for $\mathbb{B}(\mathcal{H})$ and for type III $\sigma$-finite factors, while for type II factors we characterize diagonal elements that are infinite sums of projections. (Theorem 5.11). The characterization is also complete for multiplier algebras of $\sigma$-unital purely infinite simple $\mathrm{C}^{*}$-algebras (Theorem [5.3), or more precisely for those elements in $\mathcal{M}(\mathcal{A}) \backslash \mathcal{A}$.

Finally, in Section 6, we discuss decompositions into finite sums of projections. A complete characterization is still open in $\mathbb{B}(\mathcal{H})$. We review results in this area, including the sufficient condition $\|x\|_{e}>1$ in terms of the essential norm, initially considered by Choi and $\mathrm{Wu}$ [7, (see Theorem 6.3).

We extend this condition to von Neumann factors (Theorem 6.4) and to global von Neumann algebras in terms of the central essential spectrum (Theorem 6.5).

Ideas from frame theory lead to a necessary condition in terms of operator ideals of $\mathbb{B}(\mathcal{H})$. This shows that the full characterization of finite sums of projections may require some delicate analysis.

The sufficient condition $\|x\|_{e}>1$ for $x$ to be a finite sum of projections holds also in multiplier algebras of $\sigma$-unital purely infinite simple $\mathrm{C}^{*}$-algebras (Theorem 6.8). It would thus be natural to think that positive elements of $\sigma$-unital purely infinite simple $\mathrm{C}^{*}$-algebras with operator norm greater than 1 may also be finite sums of projections. Indeed, this turns out to be the case for the Cuntz algebras $\mathcal{O}_{n}$ with $2 \leq n<\infty$ (Theorem [6.9), but at the moment we do not know if the property holds or not for $\mathcal{O}_{\infty}$. 


\section{Some facts about PROJECTIONS AND C*-ALGEBras}

During the last five decades, much research activity around operator algebras has centered at the structure of projections and their "levels of abundance". In some $\mathrm{C}^{*}$-algebras, the amount of projections can be abundant enough to give rise to an approximate spectral decomposition of all normal elements, while some other $\mathrm{C}^{*}$-algebras do not have any nontrivial projections besides the zero projection or the identity (even in certain simple $\mathrm{C}^{*}$-algebras).

Let us first recall several notions concerning the "amount" of projections in a $\mathrm{C}^{*}$-algebra.

Definition 2.1. $A C^{*}$-algebra $A$ can have the following properties:

$(\exists \mathrm{P})$ There exists a nontrivial projection in $\mathcal{A}([2])$.

(AP) $\mathcal{A}$ is generated as a $C^{*}$-algebra by its projections ([2]).

(SP) There exists a nontrivial projection in each nonzero hereditary $C^{*}$-subalgebra of $\mathcal{A}([2])$.

(LP) $\mathcal{A}$ is the closure of the span of its projections (2, 39). Equivalently, the selfadjoint part $\mathcal{A}_{\text {sa }}$ of $\mathcal{A}$ is the closure of the collection of linear combinations with real coefficients of projections in $\mathcal{A}$.

In fact, if (LP) holds and $a=a^{*} \in \mathcal{A}$, then $a=\lim \sum_{1}^{n} \lambda_{j} p_{j}$, and hence, $a=a^{*}=\lim \left(\sum_{1}^{n} \lambda_{j} p_{j}\right)^{*}$. It follows that $a=\lim \sum_{1}^{n} \frac{\lambda_{j}+\bar{\lambda}_{i}}{2} p_{j}$. The converse $i s$ also trivial.

(HLP) Every hereditary $C^{*}$-subalgebra of $\mathcal{A}$ has the LP property ([21]).

(CP) The positive part $\mathcal{A}_{+}$of $\mathcal{A}$ is the closure of all linear combinations with positive coefficients of projections of $\mathcal{A}([2])$.

(PLP) Every hereditary $C^{*}$-subalgebra of $\mathcal{A}$ has the property $C P([21])$.

(HP) Every hereditary $C^{*}$-subalgebra of $\mathcal{A}$ has an approximate identity consisting of projections ([2]).

(FS) Every self-adjoint element of $\mathcal{A}$ can be approximated in norm by self-adjoint elements with finite spectra. It is equivalent to replace "self-adjoint" by "positive" ([2]).

$(\mathrm{RR}=0)$ The collection of self-adjoint invertible elements is norm-dense in the selfadjoint part of $\mathcal{A}(\underline{6})$.

(AUD) $\mathcal{A}$ is called almost upward directed, if for any positive number $\epsilon$ and any two projections $p, q$ in $\mathcal{A}$ there exists another projection $r \in \mathcal{A}$ in the hereditary $C^{*}$-subalgebra generated by $p, q$ such that $\|(1-r) p\|<\epsilon$ and $\|(1-r) q\|<\epsilon$ ([37]).

We also recall the following facts.

\section{Remark 2.1.}

(1) The three properties $H P, F S$, and $R R=0$ are equivalent and the algebras possessing them are called of real rank zero. These are the algebras showing the most abundance of projections ([2] and [6]). And the property AUD for a multiplier algebra $M(\mathcal{A})$ is equivalent to $R R(M(\mathcal{A}))=0$ if $\mathcal{A}$ is $\sigma$-unital and of real rank zero ([37).

(2) All hereditary $C^{*}$-subalgebras of a $C^{*}$-algebra of real rank zero, including all von Neumann algebras, have the property LP ([6], [2]).

(3) All hereditary $C^{*}$-subalgebras of the multiplier algebra of a stable $\sigma$-unital $C^{*}$ algebra of real rank zero have the property LP ([39]). 
In this paper, we focus on three kinds of operator algebras: von Neumann algebras, $\sigma$-unital purely infinite simple $\mathrm{C}^{*}$-algebras, and their multiplier algebras.

Among von Neumann algebras, of main interest are of course the $\sigma$-finite factor algebras; roughly speaking, we can think of algebras operating on a separable Hilbert space. Then the (infinite) type I factors are of course identified with the algebra $\mathbb{B}(\mathcal{H})$ of bounded operators on a Hilbert space $\mathcal{H}$, the type III factors have no proper ideals nor finite nonzero projections, and the type $\mathrm{II}_{\infty}$ factors can be viewed as a continuous analogs of $\mathbb{B}(\mathcal{H})$ endowed with the ideal of "relative compact" operators.

A simple $\mathrm{C}^{*}$-algebra is called purely infinite if every hereditary $\mathrm{C}^{*}$-subalgebra of the form $\left(x \mathcal{A} x^{*}\right)^{-}$for some $x \in \mathcal{A}$ contains an infinite projection $p$ in the sense of Murray-von Neumann, namely, $p \sim p^{\prime}<p$. It is easy to see that all hereditary $\mathrm{C}^{*}$ subalgebras of a separable $\mathrm{C}^{*}$-algebra have that form. Familiar examples of such $\mathrm{C}^{*}$-algebras include all type III factors, and the Cuntz algebras $\mathcal{O}_{n}(n \leq 2 \leq \infty)$ constructed around 1980, which were the first separable examples of purely infinite simple $\mathrm{C}^{*}$-algebras.

Around 1980 both Cuntz and Blackadar were wondering about "What the abundant level of projections is in $\mathcal{O}_{n} \mathrm{~s}$ ". This question is clear from the following item (3) in terms of real rank zero.

\section{Remark 2.2.}

(1) All von Neumann algebras have real rank zero ([6]).

(2) All purely infinite simple $C^{*}$-algebras have real rank zero (35] and [38.).

(3) A simple $C^{*}$-algebra $\mathcal{A}$ is purely infinite if and only if $\mathcal{A}$ has real rank zero and every nonzero projection is infinite ([36] and [41]). Notice the analogy between these algebras and type III factors.

(4) A $\sigma$-unital purely infinite simple $C^{*}$-algebra is either unital or it is stable, i.e. $\mathcal{A} \cong \mathcal{A} \otimes \mathcal{K}([38)$.

Concerning topologies of the multiplier algebras, we recall that besides the one induced by the norm, $\mathcal{M}(\mathcal{A})$ is endowed with the strict topology defined by: $x_{\lambda} \longrightarrow$ $x$ in the strict topology if and only if

$$
\max \left\{\left\|\left(x_{\lambda}-x\right) a\right\|,\left\|\left(x_{\lambda}^{*}-x^{*}\right) a\right\|\right\} \longrightarrow 0 \quad \text { for all } a \in \mathcal{A} .
$$

If $\mathcal{A}=\mathcal{K}$, then $\mathcal{M}(\mathcal{A})=\mathbb{B}(\mathcal{H})$. In this extreme case, the strict convergence is exactly *-strong convergence (also called the double strong convergence). Thus it is natural to consider strictly converging series of projections in $\mathcal{M}(\mathcal{A})$.

The following properties illustrate the richness of the structure of $\mathcal{M}(\mathcal{A})$.

\section{Remark 2.3.}

(a) Assume that $\mathcal{A}$ is simple. Then the generalized Calkin algebra $\mathcal{N}(\mathcal{A} \otimes \mathcal{K}) / \mathcal{A} \otimes \mathcal{K}$ is simple if and only if either $\mathcal{A}$ is elementary (i.e., finite matrices or $\mathcal{K}$ ) or $\mathcal{A}$ is purely infinite ([39]).

(b) If $\mathcal{A}$ is $\sigma$-unital purely infinite and simple, then every projection in $\mathcal{N}(\mathcal{A}) \backslash \mathcal{A}$ is equivalent to the identity. (A close analogue of $\mathcal{B}(\mathcal{H})$ ) ([39]).

(c) If $\mathcal{A}$ is $\sigma$-unital purely infinite simple, then the generalized Calkin algebra $\mathcal{N}(\mathcal{A}) / \mathcal{A}$ is purely infinite and simple ([39]).

(d) Assume that $\mathcal{A}$ is $\sigma$-unital, purely infinite and simple. Then $\mathcal{M}(\mathcal{A})$ has real rank zero if and only if $K_{1}(\mathcal{A})=\{0\}$ ([38] and 39]). 


\section{Linear combinations of projections}

The structure of projections and their level of abundance have always been of central interest in the study of operator algebras. On the one hand, projections in a von Neumann algebra are so abundant to permit the spectral decomposition of all normal elements, and on the other hand, some operator algebras, even some simple unital $\mathrm{C}^{*}$-algebras, do not have any nontrivial projection at all.

In this article, we focus on algebras where every element is a linear combination of projections. It was first proven in 1967 by Fillmore that this holds for the algebra $\mathbb{B}(\mathcal{H})$ of all bounded operators on a separable Hilbert space $\mathcal{H}$.

Theorem 3.1. ([12]) If $\mathcal{H}$ is an infinite dimensional separable Hilbert space, then every operator in $\mathbb{B}(\mathcal{H})$ is a linear combination of at most 257 projections.

This estimate was subsequently improved by Pearcy and Topping [30] in the same year.

Theorem 3.2. ([30]) If $\mathcal{H}$ is an infinite dimensional separable Hilbert space, then every operator in $\mathbb{B}(\mathcal{H})$ is a linear combination of at most 16 projections.

In 1984 Matsumoto [29] showed the following best estimate in $\mathbb{B}(\mathcal{H})$ :

Theorem 3.3. (29]) If $\mathcal{H}$ is an infinite dimensional separable Hilbert space, then every selfadjoint operator in $\mathbb{B}(\mathcal{H})$ is a linear combination of 5 projections. Consequently, every operator in $\mathbb{B}(\mathcal{H})$ is a linear combination of 10 or fewer projections.

Pearcy and Topping generalized the $\mathbb{B}(\mathcal{H})$ result to infinite von Neumann factors:

Theorem 3.4. ([30]) All elements in an infinite von Neumann factor are linear combinations of projections.

The more difficult case of type $I I_{1}$ factors was settled by Fack and de La Harpe.

Theorem 3.5. (10]) All elements in a $I I_{1}$ factor are linear combinations of projections.

Thus all elements in a von Neumann factor are linear combinations of projections. Beyond factors, this property can fail. In 1992 Goldstein and Paszkiewicz proved the following:

Theorem 3.6. (14, Theorems 3.2]) A von Neumann algebra has the property that all its elements are linear combinations of projections if and only if the algebra has no finite type I direct summand with infinite-dimensional center.

In their work, Goldstein and Paszkiewicz improved the previous estimates on the number of needed projections. However, in the present article, we will not focus on those estimates, but rather on the size of the coefficients needed in the linear combinations, more precisely, on the existence of an algebra constant $v_{0}$ with the property:

Definition 3.1. A $C^{*}$-algebra $\mathcal{A}$ has a constant $v_{0}$ if every $x \in \mathcal{A}$ can be decomposed into a linear combination $x=\sum_{j=1}^{n} t_{j} p_{j}$ for certain number of projections $p_{j} \in \mathcal{A}$ (here $n$ depends on $x$ ) such that $\sum_{j=1}^{n}\left|t_{j}\right| \leq v_{o}\|x\|$.

Such a universal constant $v_{0}$ exists for every von Neumann algebra with no type I direct summand with infinite-dimensional center; the fact is implicitly given in the statements of the Goldstein and Paszkiewicz's results that are restated as follows. 
Theorem 3.7. (14, Theorems 1-3]) If a von Neumann algebra $M$ has no finite type I direct summand with infinite-dimensional center, then it has a constant $v_{o}$ as in Definition 3.1.

If $M$ is properly infinite then $v_{o} \leq 8$;

if $M$ is of type $I I_{1}$, then $v_{o} \leq 14$;

if $M$ is the direct sum of $m$ matrix algebras, then $v_{o} \leq m+4$.

The situation is of course more complicated for $\mathrm{C}^{*}$-algebras. There is a large class of $\mathrm{C}^{*}$-algebras including all unital simple AF-algebras with finitely many extreme tracial states and all unital purely infinite simple $\mathrm{C}^{*}$-algebras for which every element is equal to a linear combination of projections. We also know of the following results:

Theorem 3.8. The following $C^{*}$-algebras $\mathcal{A}$ have the property that every element is a linear combination of projections in the algebra:

(1) $\mathcal{A}$ is a unital simple real rank zero $C^{*}$-algebra with unique tracial state $\tau$ and satisfying strict comparison for projections: if $p, q \in \mathcal{A}$ are projections and $\tau(p)<\tau(q)$ then $p \prec q(27])$.

(2) $\mathcal{A}$ is a unital simple $C^{*}$-algebra with a nontrivial projection and does not admit a tracial state (27], 28]).

(3) $\mathcal{A}$ is a unital simple AT-algebra with real rank zero and finitely many extreme tracial states (26, [27]).

(4) $\mathcal{A}$ is a unital simple AH-algebra with real rank zero, bounded dimension growth and finitely many extreme tracial states such that the building blocks are finite direct sums of full matrix algebras over unital commutative $C^{*}$ algebras (with uniformly bounded dimension) (26], 27]).

Using the work of 9 ] and [26] we obtained the following

Proposition 3.9. (21, Proposition 2.6]) Let $\mathcal{A}$ be a unital $C^{*}$-algebra for which there exist two projections $p, q \in \mathcal{A}$ such that $p \sim q \sim 1$ and $p q=0$. Then there exists a universal constant $v_{0}$ as in Definition 3.1

This class of $\mathrm{C}^{*}$-algebras includes among others all unital simple infinite $\mathrm{C}^{*}$ algebras and all multiplier algebras of $\sigma$-unital stable $\mathrm{C}^{*}$-algebras.

Question 3.10. If a $C^{*}$-algebra $\mathcal{A}$ has the property that every element is a linear combination of projections in the algebra, must there exist a constant $v_{o}$ as in Definition [3.1]?

\section{Positive combinations of projections}

If in a $\mathrm{C}^{*}$-algebra all elements are linear combinations of projections, it is obvious that self-adjoint elements can be expressed as linear combinations of projections with real coefficients. What about expressing positive elements as linear combinations of projections with positive coefficients? (positive combinations of projections for short).

Fillmore remarked in 12 that positive compact operators of infinite rank cannot be decomposed as positive combinations of projections. Later in 1985, Fong and Murphy proved in [13] that these operators are the only exceptions. 
Theorem 4.1. (13]) A positive operator $x \in \mathbb{B}(\mathcal{H})$ can be written as a positive combination of projections if and only if $x$ is not a compact operator of infinite rank.

The key for obtain this result was the fact that positive invertible operators are positive combinations of projections. This fact was obtained in [11] by Fillmore from his characterization of sums of two projections (see Theorem 6.2) and again by Fong and Murphy in [13, by the existence of a constant $v_{o}$ as in Definition 3.1] The Fong and Murphy proof can be modified to prove the same result for certain $\mathrm{C}^{*}$-algebras.

Proposition 4.2. 21, Proposition 2.7] Let $\mathcal{A}$ be a unital $C^{*}$-algebra with the following two properties:

(1) $\mathcal{A}$ has a constant $v_{0}$ as in Definition [3.1;

(2) $\mathcal{A}$ satisfies condition $C P$, namely, positive combinations of projections are norm dense in $\mathcal{A}_{+}$.

Then every positive invertible element in $\mathcal{A}$ is a positive combination of projections.

The class of $\mathrm{C}^{*}$-algebras satisfying the above conditions include all von Neumann algebras with no finite type I direct summand with infinite dimensional center, all unital simple purely infinite $\mathrm{C}^{*}$-algebras, and all multiplier algebras of stable $\sigma$ unital $\mathrm{C}^{*}$-algebras of real rank zero.

The following lemma is the key to extend the positive combination property beyond invertible elements.

Lemma 4.3. (21, Lemma 2.9])

Let $\mathcal{A}$ be $C^{*}$-algebra with two mutually orthogonal projections $q \prec p$ with the property that for every projection $r \in \mathcal{A}$ such that $r \leq p$, every positive invertible element of $r \mathcal{A} r$ is a positive combination of projections in $r \mathcal{A} r$. Let $x:=t p+b$ be a positive element in $\mathcal{A}$, where $t>\|b\|$ and $b=q b=b q$. Then $x$ is a positive combination of projections in $\mathcal{A}$.

The application of this lemma to von Neumann algebras yields the following characterization of positive combinations of projections in terms of their spectral resolution.

Theorem 4.4. (20, Theorem 2.12]) Let $M$ be a von Neumann algebra, $x \in M^{+}$, $r_{x}$ be the range projection of $x$, and $\chi(x)$ be the spectral measure of $x$.

(i) Assume that $r_{x} M r_{x}$ has no finite type I direct summands with infinite dimensional center and that there is a $\delta>0$ such that $\chi_{(0, \delta)}(x) \prec \chi_{[\delta, \infty)}(x)$. Then $x$ is a positive combination of projections in $M$.

(ii) If $r_{x}$ is properly infinite, then $x$ is a positive combination of projections in $M$ if and only if there is a $\delta>0$ such that $\chi_{(0, \delta)}(x) \prec \chi_{[\delta, \infty)}(x)$.

(iii) If $r_{x} M r_{x}$ is a finite sum of finite factors or of $\sigma$-finite type III factors, then $x$ is always a positive combination of projections in $M$.

For finite algebras the spectral condition $\chi_{(0, \delta)}(x) \prec \chi_{[\delta, \infty)}(x)$ for some $\delta>0$ in Theorem 4.4(i) is only sufficient.

Question 4.5. Are there natural necessary and sufficient conditions that characterize those elements that are positive combinations of projections in a finite von Neumann algebra with infinite dimensional center? 
Notice that if $M=\mathbb{B}(\mathcal{H})$ for $\mathcal{H}$ separable and if $x$ is an infinite rank positive operator, then the condition $\chi_{(0, \delta)}(x) \prec \chi_{[\delta, \infty)}(x)$ for some $\delta>0$ holds if and only if $x$ is not compact, and the latter condition is indeed the necessary and sufficient condition in Theorem 4.1. The same equivalence holds in type $\mathrm{II}_{\infty}$ factors where in lieu of the ideal of compact operators we take the ideal $J(M)$ of relative compact operators, which has been studied first by Breuer [3, 4] and Sonis [32, i.e., the norm closed two sided-ideal generated by the finite projections of $M$. Denote by $\|\cdot\|_{e}$ the essential norm corresponding to $J(M)$, i.e., $\|x\|_{e}=\|\pi(x)\|$, where $\pi: M \rightarrow M / J(M)$ is the quotient map.

Thus the factor case is well understood and simple: either $x$ belongs to a finite factor, in which case $x$ is always a positive combination of projections or $r_{x}$ is infinite, in which case $x$ is a positive combination of projections if and only if $x \notin J(M)$, or, equivalently, $\|x\|_{e}>0$. If $M$ is of type III, then $J(M)=\{0\}$ and again $x$ is always a positive combination of projections.

In order to move beyond factors, or finite sums of factors, we need to reformulate the condition $\chi_{(0, \delta)}(x) \prec \chi_{[\delta, \infty)}(x)$ for some $\delta>0$ in terms of an appropriate ideal. The natural tool to do so is the theory of central ideals of Halpern [15, [16] and Stratila and Zsido 33, of which we will briefly review here only a couple of key notions.

A two-sided norm-closed ideal $\mathcal{J}$ of $M$ is called central if for every collection of mutually orthogonal projections $e_{\gamma} \in M \cap M^{\prime}$ and every norm-bounded family $x_{\gamma} \in \mathcal{J}$ it follows that $\sum_{\gamma} x_{\gamma} e_{\gamma} \in \mathcal{J}$ J. Given a central ideal $\mathcal{J}$ and an $x \in M$ there is a central essential spectrum of $x$ relative to $\mathcal{J}$, which is a bounded and strongly closed subset of the center. If $x \geq 0$, that subset has a maximal element $C_{u, \mathcal{J}}(x)$ which we call the central essential norm of $x$.

For instance, if $M=\bigoplus_{k=1}^{\infty} \mathbb{B}\left(\mathcal{H}_{k}\right)$ with $\mathcal{H}_{k} \equiv \mathcal{H}, \mathcal{J}=\bigoplus_{k=1}^{\infty} K\left(\mathcal{H}_{k}\right)$ and $x=$ $\bigoplus_{k=1}^{\infty} x_{k} \in M^{+}$, then $\mathcal{J}$ is a central ideal of $M$ and $C_{u, \mathcal{J}}(x)=\bigoplus_{k=1}^{\infty}\left\|x_{k}\right\|_{e} I_{k}$ where $\left\|x_{k}\right\|_{e}$ is the essential norm of $x_{k}$.

Given a properly infinite projection $p \in M$, there is an associated central ideal $J(p)$ of "dimension less than $p$ ", namely the ideal whose set of projections is

$$
\left\{q \in M \mid q \leq c(p) \text { and } p g \prec q g \text { for some projection } g \in M \cap M^{\prime} \Rightarrow p g=0 .\right\}
$$

where $c(p)$ denotes the central support of $p$.

While in an infinite factor the necessary and sufficient condition for a positive element $x$ to be a positive combination of projections is that its essential norm is nonzero, for global algebras we must require that the central essential norm be bounded away from zero. More precisely:

Theorem 4.6. (20, Theorem 3.4]) Let $M$ be a von Neumann algebra, let $x \in M^{+}$ and assume that $r_{x}$ is properly infinite. Set $\mathcal{J}=\mathcal{J}\left(r_{x}\right)$. Then $T$ is a positive combination of projections in $M$ if and only if $C_{u, \mathcal{J}}(x)$ is locally invertible, i.e., there is a $\nu>0$ for which $C_{u, \mathcal{J}}(x) \geq \nu c\left(r_{x}\right)$.

It is often easier in properly infinite von Neumann algebras to decompose elements into linear combinations of projections, positive combinations of projections, or sums of projections, and and to investigate commutators than in the finite von Neuamnn algeras.

Thus in moving now our focus to $\mathrm{C}^{*}$-algebras, we naturally start with $\sigma$-unital purely infinite simple $\mathrm{C}^{*}$-algebras and their associated multiplier algebras. Both 
these kinds of algebras satisfy the conditions for Proposition 4.2 and Lemma 4.3 and Lemma 4.3 plays again a key role.

Perhaps not surprisingly the situation for $\sigma$-unital purely infinite simple $\mathrm{C}^{*}$ algebras is similar to the one for type III factors:

Theorem 4.7. 21, Theorem 2.11] Let $\mathcal{A}$ be a $\sigma$-unital purely infinite simple $C^{*}$ algebra. Then every nonzero positive element of $\mathcal{A}$ is a positive combination of projections.

The crux of the proof is the case when the hereditary algebra generated by $x$ is unital and the number $\|t\|$ is not an isolated point in the spectrum of $x$ and then the proof consists of a reduction to the situation in Lemma 4.3. In the general case, the hereditary algebra generated by $x$ can be embedded in a unital hereditary subalgebra of $\mathcal{A}$ and then the result also follows.

A similar proof combined with results for $\mathbb{B}(\mathcal{H})$ from 7 and [20, shows that if $x \in \mathcal{M}(\mathcal{A})^{+} \backslash \mathcal{A}$ then $x$ is a scalar multiple of a finite sum of projections. Here, the analogy is to the case when $x \in \mathbb{B}(\mathcal{H})^{+} \backslash K(\mathcal{H})$. But then, combining this result with the one of Theorem 4.7, we get:

Theorem 4.8. (21, Theorem 3.1]) Let $\mathcal{A}$ be a $\sigma$-unital purely infinite simple $C^{*}$ algebra. Then every nonzero positive element of $\mathcal{M}(\mathcal{A})$ is a positive combination of projections.

\section{Infinite sums of projections}

A motivation for studying infinite sums of projections in $\mathbb{B}(\mathcal{H})$ with convergence in the strong topology strong sums for short, comes from frame theory. Indeed Question 1.3 for $\mathbb{B}(\mathcal{H})$ is reformulation of the frame theory question of which positive operators are the Bessel operators of a uniform-norm Bessel sequence. With this motivation, Dykema, Freeman, Kornelson, Larson, Ordower and Weber in [8] investigated Question 1.3 and found that a sufficient condition for a positive operator $x$ to be a sum of projections is that the essential norm $\|x\|_{e}>1$. A more general question - 'which positive operators are the Bessel operators of a Bessel sequence with pre-assigned norms' - was studied by Kornelson and Larson in 2004 ([22]), and by Antezana, Massey, Ruiz and Stojanoff in 2007 ([1]), leading to an alternative approach to the Schur-Horn Theorem.

A full answer to Question 1.3 was recently obtained by the authors of the present article for the case when $M$ is a $\sigma$-finite type I or type III von Neumann factor, and for diagonalizable operators in the case when $M$ is a $\sigma$-finite type II factor. (An operator $x \in M^{+}$is diagonalizable if $x=\sum \gamma_{j} e_{j}$ for some $\gamma_{j}>0$ and mutually orthogonal projections $e_{j} \in M$.) The characterization of strong sums of projections is given in terms of the notions of excess part and defect part of an operator defined as follows:

$$
\begin{aligned}
& \chi \\
& r_{x}=\chi_{(0, \| x||]}(x) \\
& x_{+}:=(x-I) \chi_{(1,\|x\|]}(x) \\
& x_{-}:=(I-x) \chi_{(0,1)}(x)
\end{aligned}
$$

the characteristic function

the range projection of $x$

the excess part of $x$

the defect part of $x$.

Thus we have the decomposition

$$
x=x_{+}-x_{-}+r_{x} .
$$


Notice that $\|x\|_{e}>1$ if and only if $x_{+}$is not compact.

Theorem 5.1. (17, Theorem 1.1])

(i) Let $M=\mathbb{B}(\mathcal{H})$ and $x \in M^{+}$. Then $x$ is a strong sum of projections if and only if either $\operatorname{Tr}\left(x_{+}\right)=\infty$ or $\operatorname{Tr}\left(x_{-}\right) \leq \operatorname{Tr}\left(x_{+}\right)<\infty$ and $\operatorname{Tr}\left(x_{+}\right)-\operatorname{Tr}\left(x_{-}\right) \in \mathbb{N} \cup\{0\}$.

(ii) Let $M$ be a $\sigma$-finite type II factor, $\tau$ be a faithful, semifinite, normal trace on $M^{+}$, and $x \in M^{+}$. If $x$ is a strong sum of projections, then $\tau\left(x_{+}\right) \geq \tau\left(x_{-}\right)$. If either $\tau\left(x_{+}\right)=\infty$ or $x$ is diagonalizable and $\tau\left(x_{+}\right) \geq \tau\left(x_{-}\right)$, then $T$ is a strong sum of projections.

(iii) Let $M$ be a $\sigma$-finite type III factor and $x \in M^{+}$. Then $x$ is a strong sum of projections if and only if either $\|x\|>1$ or $x$ is a projection.

The proof is based on a $2 \times 2$ matrix construction that decomposes linear combinations $(1-\lambda) p_{1}+(1+\mu) p_{2}$ of two equivalent projections $p_{1}$ and $p_{2}$, with $\lambda, \mu>0$, $\lambda \leq 1$, into the combination $q_{1}+(1+\mu-\lambda) q_{2}$ of two equivalent projections $q_{1}$ and $q_{2}$. A reduction to strong sums of such linear combinations is always possible in the type I or type III case, or in the type II case when $\tau\left(x_{+}\right)=\infty$. To achieve this reduction in the type II case when $\tau\left(x_{+}\right)<\infty$, we ask for $x$ to be diagonalizable. However, it is easy to find examples of strong sums of projections in type $\mathrm{II}_{1}$ factors, even sums of two projections, that are not diagonalizable. This leads us to ask:

Question 5.2. Can the hypothesis that $x$ is diagonalizable be removed from (ii)?

In many respects, the strict topology in the multiplier algebra $\mathcal{M}(\mathcal{A})$ of a $\mathrm{C}^{*}$ algebra $\mathcal{A}$ takes the role of the strong topology in von Neumann factors. Multiplier algebras have also a natural notion of essential norm, namely for every $x \in \mathcal{M}(\mathcal{A})$, we denote by $\|x\|_{e}$ the norm of $\pi(x)$ where $\pi: \mathcal{M}(\mathcal{A}) \rightarrow \mathcal{M}(\mathcal{A}) / \mathcal{A}$ is the canonical quotient map. When $\mathcal{A}$ is sufficiently "nice", which in this context is $\sigma$-unital, nonunital, purely infinite simple, we have the following characterization of those elements that are infinite sums of projections.

Theorem 5.3. (19, Theorem 1.1 ]) Let $\mathcal{A}$ be a $\sigma$-unital, nonunital, purely infinite simple $C^{*}$-algebra and $x$ be a positive element of $\mathcal{M}(\mathcal{A})$. Then $x$ is a strictly converging sum of projections belonging to $\mathcal{A}$ if and only if one of the following mutually exclusive conditions hold:

(i) $\|x\|_{e}>1$.

(ii) $\|x\|_{e}=1$ and $\|x\|>1$.

(iii) $x \in \mathcal{M}(\mathcal{A}) \backslash \mathcal{A}$ is a projection.

(iv) $x$ is the sum of finitely many projections belonging to $\mathcal{A}$.

A key step in the proof of this theorem, whose crux is case (ii), and a result of independent interest in itself, is the following:

Lemma 5.4. (19, Lemma 2.5]) For every $\sigma$-unital, nonunital, purely infinite simple $C^{*}$-algebra $\mathcal{A}$ the collection of finite sums of projections is norm dense in $\left\{x \in \mathcal{A}^{+} \mid\|x\|>1\right\}$.

Notice that case (iv) in Theorem 5.3 leads naturally to the harder Question 1.2 (see the next section).

\section{Finite sums of projections}

The question of which positive operators are finite sums of projections, i.e., Question 1.2 is harder, and indeed it is still open in $\mathbb{B}(\mathcal{H})$. 
The first results in this direction were obtained in 1969 by Fillmore:

Theorem 6.1. [1] A finite rank positive operator $x \in \mathbb{B}(\mathcal{H})$ is a sum of projections if and only if $\operatorname{Tr}(x) \in \mathbb{N}$ and $\operatorname{Tr}(x) \geq \operatorname{rank}(x)$.

Theorem 6.2. [1] A positive operator $x \in \mathbb{B}(\mathcal{H})$ is a sum of two projections if and only if $x=2 p \oplus b$ where $p$ is a possibly zero projection and $b$ is a positive operator unitarily equivalent to $2 I-b$.

In 1988, Choi and Wu obtained the following result that was announced in a survey article [34, Theorem 4.12] by $\mathrm{Wu}$ and was presented together with other related results in their recent paper [7, Theorem 2.2].

Theorem 6.3. 7] A sufficient condition for a positive operator $x \in \mathbb{B}(\mathcal{H})$ to be a finite sum of projections is that the essential norm $\|x\|_{e}>1$.

A special case of this result, i.e., for $x=\alpha I$ was obtained through completely different methods by Kruglyak, Rabanovich, and Samoilenko (see [23], 24], and several other articles referenced there) who characterized the values of $\alpha>1$ for which $\alpha I$ is the sum of $n$ or more projections.

The authors together with Halpern recently re-obtained independently the Choi and $\mathrm{Wu}$ sufficient condition in the more general setting of von Neumann algebras. For $\sigma$-finite factors the formulation is very similar to the $\mathbb{B}(\mathcal{H})$ one.

Theorem 6.4. 20, Corollary 4.4] Let $M$ be an infinite $\sigma$-finite factor and $x \in$ $M^{+}$. A sufficient condition for $x$ to be a finite sum of projections in $M$ is that

(i) $\|x\|_{e}>1$ when $M$ is of type $I_{\infty}$ (usual essential norm of $\mathbb{B}(\mathcal{H})$ );

(ii) $\|x\|_{e}>1$ when $M$ is of type $I I_{\infty}$ (essential norm relative to the ideal $J(M)$ generated by the finite projections of $M)$;

(iii) $\|x\|>1$ when $M$ is of type III (operator norm).

The proof is based on decomposing the operator into a sum of finite rank summands where the range projections of non-consecutive summands are orthogonal, and where each summand is a finite sum of projections, with the number of projections needed for each summand being uniformly bounded.

Naturally, for global properly infinite von Neumann algebras the essential norm has to be replaced by the central essential norm $C_{u, \mathcal{J}}(x)$ with respect to the central ideal $\mathcal{J}\left(r_{x}\right)$ with "dimension less than" $r_{x}$ (see Section 4.)

Theorem 6.5. 20, Theorem 4.3] Let $M$ be a von Neumann algebra. Assume that $x \in M^{+}$has a properly infinite range projection $r_{x}$ and $C_{u, \mathcal{J}}(x) \geq \nu c\left(r_{x}\right)$ for some $\nu>1$. Then $x$ is a finite sum of projections in $M$.

For the case of type $\mathrm{II}_{1}$ factors we have a sufficient condition in terms of the excess and defect part of the operator, albeit again only for the case of diagonalizable operators, (cf. Theorem 5.1).

Theorem 6.6. 20, Theorem 6.5] Let $M$ be a type $I I_{1}$ factor and let $x \in M$ be a positive diagonalizable operator. Then a sufficient condition for $x$ to be a finite sum of projections in $M$ is that $\tau\left(x_{+}\right)>\tau\left(\left(x_{-}\right)\right.$.

It is easy to see that the condition that the essential norm $\|x\|_{e}>1$ is not necessary for $x$ to be a finite sum of projections. In fact, a necessary condition, which for simplicity's sake we formulate just for $\mathbb{B}(\mathcal{H})$, is 
Theorem 6.7. (20, Corollary 5.7] Let $x \in \mathbb{B}(\mathcal{H})^{+}$be a finite sum of projections and assume that $x_{+} \in K(H)$. Then $x_{-} \in K(H)$. If $x_{-}=0$ then $x_{+}$has finite rank. If $x_{-} \neq 0$ then $x_{+}$and $x_{-}$generate the same two-sided ideal of $\mathbb{B}(\mathcal{H})$.

This necessary condition permits to easily construct examples of positive operators (with essential norm $=1$ ), that are strong sums of projections but cannot be finite sums of projections.

In passing now to $\mathrm{C}^{*}$-algebras, we notice that the same proof that permits to decompose a positive element $x \in \mathcal{N}(\mathcal{A}) \backslash \mathcal{A}$ into a positive combination of projections (see Theorem 4.8) actually guarantees that if $\|x\|_{e}>1$, then one of the coefficients $t_{j}>1$ and thus reduce the problem to the case that $x=t p \oplus s q$ where $t>1$ and $s \geq 0$. By considering $p$ and $q$ as infinite projections in a copy of $\mathbb{B}(\mathcal{H})$ embedded unitally in a corner of $\mathcal{M}(\mathcal{A})$, this reduces the problem to $\mathbb{B}(\mathcal{H})$ :

Theorem 6.8. (21, Theorem 3.3]) Let $\mathcal{A}$ be a $\sigma$-unital but nonunital purely infinite simple $C^{*}$-algebra. Then every positive element $x \in \mathcal{M}(\mathcal{A}) \backslash \mathcal{A}$ with $\|x\|_{e}>1$ is a finite sum of projections. Consequently, every nonzero positive element of $\mathcal{M}(\mathcal{A}) \backslash \mathcal{A}$ can be written as a positive scalar multiple of a sum of projections.

The problem when $\|x\|_{e}=0$, i.e., $x \in \mathcal{A}$ seems harder. So far we have obtained this result which did surprise us:

Theorem 6.9. If $2 \leq n<\infty$, then every positive element $x$ in the Cuntz algebra $\mathcal{O}_{n}$ with $\|x\|>1$ is a sum of finitely many projections.

\section{ACKNOWLEDGEMENTS}

S. Zhang would like to acknowledge that his research was partially supported by Taft International Travel Grant. P. W. Ng would like to acknowledge that his research was done while visiting the other two authors at the University of Cincinnati, in trips supported by the UC Math Department.

\section{REFERENCES}

[1] J. Antezana, P. Massey, M. Ruiz and D. Stojanoff, The Schur-Horn Theorem for operators and frames with prescribed norms and frame operator Illinois J. of Math., 51 (2007) no. 2, 537-560

[2] B. Blackadar, Notes on the structure of projections in simple algebras, Semesterbericht Funktionalanalysis, Tubingen, Wintersemester (1982/83)

[3] M. Breuer, Fredholm theories in von Neumann algebras. I Math. Ann., 178 (1968) 243-254.

[4] M. Breuer, Fredholm theories in von Neumann algebras. II Math. Ann., 180 (1969) 313-325.

[5] L.G. Brown, Stable isomorphism of hereditary subalgebras of $C^{*}$-algebras Pacific J. Math., 71 (1977) 335-348

[6] L. Brown and G. Pedersen, $C^{*}$-algebras of real rank zero J. Funct. Anal., 99 (1991) 131-149

[7] M. D. Choi and P. Y. Wu, Sums of orthogonal projections Preprint.

[8] K. Dykema, D. Freeman, K Kornelson, D. Larson, M. Ordower and E. Weber, Ellipsoidal tight frames and projection decompositions of operators Illinois J. Math., 48 (2004) no. 2, 477-489

[9] T. Fack, Finite sums of commutators in $C^{*}$-algebras Ann. Inst. Fourier (Grenoble), 32 (1982) no. 1, 129-137

[10] T. Fack and P. de la Harpe, Sommes de commutateurs dans les algebres de von Neumann finies continues Ann. Inst. Fourier (Grenoble), 30 (1980) no. 3, 49-73

[11] P. Fillmore, On sums of projections J. Funct. Anal., 4 (1969) 146-152

[12] P. Fillmore, Sums of operators with square zero Acta Sci. Math. (Szeged), 28 (1967) 285-288 
[13] C. K. Fong and G. J. Murphy, Averages of projections J. Operator Theory, 13 (1985) no. $2,219-225$

[14] S. Goldstein, and A. Paskiewicz, Linear combinations of projections in von Neumann algebras Proc. Amer. Math. Soc., 116 ( 1992) no. 1, 175-183

[15] H. Halpern, Essential central spectrum and range for elements of a von Neumann algebra Pacific J. Math., 43 (1972) no. 2, 349-380.

[16] H. Halpern, Essential Central Range and selfadjoint commutators in properly infinite von Neumann algebras Trans. AMS., 228 (1977) 117-146.

[17] V. Kaftal and P. W. Ng and S. Zhang, Strong sums of projections in von Neumann factors Journal of Functional Analysis, 257 (2009) no. 8, 2497-2529

[18] V. Kaftal and P. W. Ng and S. Zhang. Finite sums of projections in purely infinite simple $C^{*}$-algebras with torsion $K_{0}$. Proceedings AMS. To appear.

[19] V. Kaftal and P. W. Ng and S. Zhang, Projection decomposition in multiplier algebras Math Ann., (2009) To appear

[20] V. Kaftal, P. W. Ng and S. Zhang, Finite sums of projections in von Neumann algebras , (2010) preprint

[21] V. Kaftal, P. W. Ng, and S. Zhang, Positive combinations and sums of projections in purely infinite simple $C^{*}$-algebras and their multiplier algebras Proceedings AMS, 139, No 8, (2011), $2735-2746$.

[22] K. Kornelson and D. Larson, Rank-one decomposition of operators and construction of frames Contemp. Math., 345 (2004) 203-214

[23] S. Kruglyak, V. Rabanovich and Y. Samoilenko, On Sums of Projections Funct Anal and Appl., 36 (2002) 182-195

[24] S. Kruglyak, V. Rabanovich and Y. Samoülenko, Decomposition of a scalar matrix into a sum of orthogonal projections. Lin. Alg and Applic., 370 (2003) 217-225

[25] H. Lin and S. Zhang, On infinite simple $C^{*}$-algebras J. Funct. Anal., 100 (1991) no. 1, 221-231

[26] L. W. Marcoux, On the linear span of projections in certain simple $C^{*}$-algebras Indiana Univ. Math. J., 51 (2002) no. 3, 753-771

[27] L. W. Marcoux, Sums of small number of commutators J. Operator Theory, 56 (2006) no. $1,111-142$

[28] L. W. Marcoux and G. J. Murphy, Unitarily-invariant linear subspaces in $C^{*}$-algebras Proc. Amer. Math. Soc., 126 (1998) 3597-3605

[29] K. Matsumoto, Selfadjoint operators as a real span of 5 projections Math. Japon., 29 (1984) 291-294

[30] C. Pearcy and D. Topping, Sums of small numbers of idempotents Michigan Math. J., 14 (1967) 453-465

[31] G. K. Pedersen, The linear span of projections in simple $C^{*}$-algebras J. Operator Theory, 4 (1980) no. 2, 289-296

[32] M.G. Sonis, On a class of operators in von Neumann algebras with Segal measures Math USSR Sbornik, 13 (1971) no. 3, 344-359

[33] S. Stratila and L. Zsido, An algebraic reduction theory for $W^{*}$-algebras. II. Rev. Roumaine Math. Pures Appl., 18 (1973) 407-460.

[34] P. Y. Wu, Additive combinations of special operators Funct. Anal Oper. Theory. Banach Ctr Publ, Inst Math Polish Acad Sci., 30 (1994) 337-361.

[35] S. Zhang, On the structure of projections and ideals of corona algebras Canad. J. Math., 41 (1989) no. 4, 721-742

[36] S. Zhang, A property of purely infinite simple $C^{*}$-algebras Proc. Amer. Math. Soc., 109 (1990) no. 3, 717-720

[37] S. Zhang, $C^{*}$-algebras with real rank zero and the internal structure of their corona and multiplier algebras. III Canad. J. Math., 42 (1990) no. 1, 159-190

[38] S. Zhang, $C^{*}$-algebras with real rank zero and the internal structure of their corona and multiplier algebras. II K-Theory, 6 (1992) 1-27 
[39] S. Zhang, A Riesz decomposition property and ideal structure of multiplier algebras J. Operator Theory, 24 (1990) no. 2, 209-225

[40] S. Zhang, Matricial structure and homotopy type of simple $C^{*}$-algebras with real rank zero J. Operator Theory, 26 (1991) 283-312

[41] S. Zhang, Certain $C^{*}$-algebras with real rank zero and their corona and multiplier algebras. I Pacific J. Math., 155 (1992) no. 1, 169-197

Department of Mathematics, University of Cincinnati, P. O. Box 210025, Cincinnati, $\mathrm{OH}, 45221-0025$, USA

E-mail address: victor.kaftal@math.uc.edu

Department of Mathematics, University of Louisiana, 217 Maxim D. Doucet Hall, P.O. Box 41010, LAFAYETTE, Louisiana, 70504-1010, USA

E-mail address: png@louisiana.edu

Department of Mathematics, University of Cincinnati, P.O. Box 210025, Cincinnati, $\mathrm{OH}, 45221-0025$, USA

E-mail address: zhangs@math.uc.edu 